International Journal of Pure and Applied Mathematics

Volume 87 No. 3 2013, 397-403

ISSN: 1311-8080 (printed version); ISSN: 1314-3395 (on-line version)

url: http://www.ijpam.eu

doi: http://dx.doi.org/10.12732/ijpam.v87i3.3

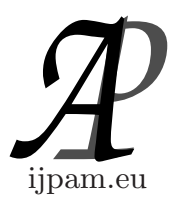

\title{
SHORT NOTE ON THE DISTRIBUTIONAL DIFFRACTION FRESNEL SINE (COSINE) TRANSFORM
}

\author{
S.K.Q. Al-Omari \\ Department of Applied Sciences \\ Faculty of Engineering Technology \\ Al-Balqa' Applied University \\ Amman 11134, JORDAN
}

\begin{abstract}
The diffraction Fresnel sine and diffraction Fresnel cosine transforms are extended to spaces of distributions of compact support. The convolution Theorem of the transforms has been established in a generalized sense. Certain theorems are also discussed.
\end{abstract}

AMS Subject Classification: 54C40, 14E20, 46E25, 20C20

Key Words: diffraction Fresnel integral, diffraction Fresnel sine transform, diffraction Fresnel cosine transform, distribution space

\section{Introduction}

The diffraction Fresnel transform of a function $f(t)$ is defined by [5]

$$
\mathrm{F}_{d} f(\xi)=\int_{\mathrm{R}} K\left(\alpha_{1}, \gamma_{1}, \gamma_{2}, \alpha_{2} ; \xi, t\right) f(t) d t
$$

$K\left(\alpha_{1}, \gamma_{1}, \gamma_{2}, \alpha_{2} ; \xi, t\right)$ being the transform kernel function given by

$$
K\left(\alpha_{1}, \gamma_{1}, \gamma_{2}, \alpha_{2} ; \xi, t\right)=\frac{1}{\sqrt{2 \pi i \gamma_{1}}} \exp \left(\frac{i}{2 \gamma_{1}}\left(\alpha_{1} t^{2}-2 \xi t+\alpha_{2} \xi^{2}\right)\right)
$$

where the real parameters, $\alpha_{1}, \gamma_{1}, \gamma_{2}$ and the prarameter $\alpha_{2}$ are defined so that 
$\alpha_{1} \alpha_{2}-\gamma_{1} \gamma_{2}=1$

If the parameters $\alpha_{1}, \gamma_{1}, \gamma_{2}$ and $\alpha_{2}$ are related by the matrix

$$
\left(\begin{array}{cc}
\alpha_{1} & \gamma_{1} \\
\gamma_{2} & \alpha_{2}
\end{array}\right)=\left(\begin{array}{cc}
\cos \theta & \sin \theta \\
-\sin \theta & \cos \theta
\end{array}\right)
$$

then the diffraction Fresnel transform becomes a fractional Fourier transform.

Let $\mathcal{S}$ denote the space of all complex valued functions $\phi(t)$ that are infinitely smooth and satisfies the infinite set of inequalities

$$
\left|t^{m} \phi^{(k)}(t)\right| \leq C_{m, k}, t \in \mathrm{R},
$$

where $m$ and $k$ traverse the set of nonegative integers [5, (2.1)]. Members of $\mathcal{S}$ are testing functions of rapid descent. The strong dual $\mathcal{S}^{\prime}$ of $\mathcal{S}$ defines a space of distributions of slow growth (the space of tempered distributions). See $[7,6,4]$.

The Parseval's relation of the diffraction Fresnel transform was established in $[5$, Theorem 2.2] as

$$
\int_{\mathrm{R}} f(x) \mathrm{F}_{d} g(x) d x=\int_{\mathrm{R}} \mathrm{F}_{d} f(x) g(x) d x
$$

where $\mathrm{F}_{d} g$ and $\mathrm{F}_{d} f$ are the respective diffraction Fresnel transforms of $f$ and $g$.

\section{Distribution Spaces and $\mathrm{F}_{d}$ Analysis}

When discussing the distribution spaces $\mathcal{S}^{\prime}$; see [7], the extended transform $\overrightarrow{\mathrm{F}}_{d}$ of a slow growth distribution $f \in \mathcal{S}^{\prime}$ is expressed as [5, (2.10)]

$$
\left\langle\overrightarrow{\mathrm{F}}_{d} f, \phi\right\rangle=\left\langle f, \mathrm{~F}_{d} \phi\right\rangle
$$

for every $\phi \in \mathcal{S}$.

Let $f \in \mathcal{E}^{\prime}$ (the space of distributions of compact supports) then the distributional transform of $f$ is extended in the same citation as

$$
\overrightarrow{\mathrm{F}}_{d} f(\xi)=\frac{1}{\sqrt{2 \pi i \gamma_{1}}}\left\langle f(t), \exp \left(\frac{i\left(\alpha_{1} t^{2}-2 t \xi+\alpha_{2} \xi^{2}\right)}{2 \gamma_{1}}\right)\right\rangle ;
$$

see $[5,(2.10)]$.

In this note, let (1) be factored into the components:

$$
\mathrm{F}_{d} f(\xi)=\mathrm{F}_{c} f(\xi)+i \mathrm{~F}_{s} f(\xi)
$$


where

$$
\mathrm{F}_{s} f(\xi)=\frac{e^{\frac{i \alpha_{2} \xi^{2}}{2 \gamma_{1}}}}{\sqrt{2 \pi i \gamma_{1}}} \int_{\mathrm{R}} f(t) \sin \left(\frac{\alpha_{1} t^{2}-2 \xi t}{2 \gamma_{1}}\right) d t
$$

and

$$
\mathrm{F}_{c} f(\xi)=\frac{e^{\frac{i \alpha_{2} \xi^{2}}{2 \gamma_{1}}}}{\sqrt{2 \pi i \gamma_{1}}} \int_{\mathrm{R}} f(t) \cos \left(\frac{\alpha_{1} t^{2}-2 \xi t}{2 \gamma_{1}}\right) d t .
$$

then the integral equations (5) and (6) interpretted to present a diffraction Fresnel sine and diffraction Fresnel cosine transforms, respectively.

From (4) we have the following definition :

Definition 2.1. Let $f \in \mathcal{E}^{\prime}(\mathrm{R})$ then the extended diffraction Fresnel sine and diffraction Fresnel cosine transforms of $f$ are defined respectively as

$$
\overrightarrow{\mathrm{F}_{s}} f(\xi)=e^{\frac{i \alpha_{2} \xi^{2}}{2 \gamma_{1}}}\left\langle f(t), \sin \frac{\left(\alpha_{1} t^{2}-2 \xi t\right)}{2 \gamma_{1}}\right\rangle,
$$

and

$$
\overrightarrow{\mathrm{F}}_{c} f(\xi)=e^{\frac{i \alpha_{2} \xi^{2}}{2 \gamma_{1}}}\left\langle f(t), \cos \frac{\left(\alpha_{1} t^{2}-2 \xi t\right)}{2 \gamma_{1}}\right\rangle .
$$

Analyticity of $\overrightarrow{\mathrm{F}_{s}}$ and $\overrightarrow{\mathrm{F}_{c}}$ can be expressed to mean :

Theorem 2.2. Let $f \in \mathcal{E}^{\prime}(\mathrm{R})$ then $\overrightarrow{\mathrm{F}_{s}}$ and $\overrightarrow{\mathrm{F}_{c}}$ are analytic and

$$
\mathcal{D}_{\xi} \overrightarrow{\mathrm{F}_{s}} f(\xi)=\left\langle f(t), \mathcal{D}_{\xi} e^{\frac{i \alpha_{2} \xi^{2}}{2 \gamma_{1}}} \sin \left(\frac{\alpha_{1} t^{2}-2 \xi t}{2 \gamma_{1}}\right)\right\rangle
$$

and

$$
\mathcal{D}_{\xi} \overrightarrow{\mathrm{F}}_{c} f(\xi)=\left\langle f(t), \mathcal{D}_{\xi} e^{\frac{i \alpha_{2} \xi^{2}}{2 \gamma_{1}}} \cos \left(\frac{\alpha_{1} t^{2}-2 \xi t}{2 \gamma_{1}}\right)\right\rangle .
$$

Proof of this theorem is analogous to that found in the literature; see the corresponding theorem in [5]

Theorem 2.3. The transforms (7) and (8) are linear.

The proof of theorem follows from simple computations in $\mathcal{E}^{\prime}$.

Let $f$ and $g$ be in $\mathcal{E}^{\prime}$ then the generalized convolution of $f$ and $g$ is defined by $[7, \mathrm{P} .123,(2)]$

$$
\langle f * g, \phi\rangle=\langle f(t) \times g(\tau), \phi(t+\tau)\rangle=\langle f(t),\langle g(\tau), \phi(t+\tau)\rangle\rangle .
$$


We have the following theorem.

Theorem 2.4. (Convolution Theorem) Let $f \in \mathcal{E}^{\prime}, g \in \mathcal{E}^{\prime}$ then

$$
\overrightarrow{\mathrm{F}_{s}}(f * g)(s)=\left\langle f(t) \sin \frac{s t}{\gamma_{1}}, \overrightarrow{\mathrm{F}_{c}}(g(x))(t)\right\rangle+\left\langle f(t) \cos \frac{s t}{\gamma_{1}}, \overrightarrow{\mathrm{F}_{s}}(g(x))(t)\right\rangle .
$$

Proof. Since the diffraction Fresnel sine transform is defined by

$$
\left(\mathrm{F}_{s} f\right)(s)=\frac{e^{\frac{i}{2}\left(\frac{\alpha_{2}}{\gamma_{1}}\right) s^{2}}}{\sqrt{2 \pi i \gamma_{1}}} \int_{\mathrm{R}} \sin \left(\frac{s}{\gamma_{1}} t\right) e^{\frac{i}{2}\left(\frac{\alpha_{1}}{\gamma_{1}}\right) t^{2}} f(t) d t
$$

it acts on $*$ as

$$
\begin{aligned}
\overrightarrow{\mathrm{F}}_{s}(f * g)(s) & =\frac{e^{\frac{i}{2}\left(\frac{\alpha_{2}}{\gamma_{1}}\right) s^{2}}}{\sqrt{2 \pi i \gamma_{1}}}\left\langle f * g(t), e^{\frac{i}{2}\left(\frac{\alpha_{1}}{\gamma_{1}}\right) t^{2}} \sin \left(\frac{s}{\gamma_{1}} t\right)\right\rangle \\
& =\frac{e^{\frac{i}{2}\left(\frac{\alpha_{2}}{\gamma_{1}}\right) s^{2}}}{\sqrt{2 \pi i \gamma_{1}}}\left\langle f(t),\left\langle g(x), e^{\frac{i}{2}\left(\frac{\alpha_{1}}{\gamma_{1}}\right)(t+x)^{2}} \sin \left(\frac{s}{\gamma_{1}}(t+x)\right)\right\rangle\right\rangle .
\end{aligned}
$$

Using the fact that

$$
\sin \left(\frac{s t}{\gamma_{1}}+\frac{s x}{\gamma_{1}}\right)=\sin \frac{s t}{\gamma_{1}} \cos \frac{s x}{\gamma_{1}}+\sin \frac{s x}{\gamma_{1}} \cos \frac{s t}{\gamma_{1}}
$$

we get

$$
\begin{aligned}
\overrightarrow{\mathrm{F}_{s}} & (f * g)(s)=\frac{e^{\frac{i}{2}\left(\frac{\alpha_{2}}{\gamma_{1}}\right) s^{2}}}{\sqrt{2 \pi i \gamma_{1}}} \times \\
& \left\langle f(t),\left\langle g(x), e^{\frac{i}{2}\left(\frac{\alpha_{1}}{\gamma_{1}}\right)(t+x)^{2}}\left(\sin \frac{s t}{\gamma_{1}} \cos \frac{s x}{\gamma_{1}}+\sin \frac{s x}{\gamma_{1}} \cos \frac{s t}{\gamma_{1}}\right)\right\rangle\right\rangle \\
= & \frac{e^{\frac{i}{2}\left(\frac{\alpha_{2}}{\gamma_{1}}\right) s^{2}}}{\sqrt{2 \pi i \gamma_{1}}}\left\langle f(t),\left\langle g(x), e^{\frac{i}{2}\left(\frac{\alpha_{1}}{\gamma_{1}}\right)(t+x)^{2}} \sin \frac{s t}{\gamma_{1}} \cos \frac{s x}{\gamma_{1}}\right\rangle\right\rangle \\
& +\frac{e^{\frac{i}{2}\left(\frac{\alpha_{2}}{\gamma_{1}}\right) s^{2}}}{\sqrt{2 \pi i \gamma_{1}}}\left\langle f(t),\left\langle g(x), e^{\frac{i}{2}\left(\frac{\alpha_{1}}{\gamma_{1}}\right)(t+x)^{2}} \sin \frac{s x}{\gamma_{1}} \cos \frac{s t}{\gamma_{1}}\right\rangle\right\rangle \\
= & \left\langle e^{\frac{i}{2}\left(\frac{\alpha_{1}}{\gamma_{1}}\right) t^{2}} f(t) \sin \frac{s t}{\gamma_{1}},\left\langle e^{\frac{i}{2}\left(\frac{\alpha_{1}}{\gamma_{1}}\right)(2 t x)} g(x), \frac{e^{\frac{i}{2}\left(\frac{\alpha_{2}}{\gamma_{1}}\right) s^{2}} e^{\frac{i}{2}\left(\frac{\alpha_{2}}{\gamma_{1}}\right) x^{2}}}{\sqrt{2 \pi i \gamma_{1}}} \cos \frac{s x}{\gamma_{1}}\right\rangle\right\rangle \\
& +\left\langle e^{\frac{i}{2}\left(\frac{\alpha_{1}}{\gamma_{1}}\right) t^{2}} f(t) \cos \frac{s t}{\gamma_{1}},\left\langle e^{\frac{i}{2}\left(\frac{\alpha_{1}}{\gamma_{1}}\right)(2 t x)} g(x), \frac{e^{\frac{i}{2}\left(\frac{\alpha_{2}}{\gamma_{1}}\right) s^{2}} e^{\frac{i}{2}\left(\frac{\alpha_{2}}{\gamma_{1}}\right) x^{2}}}{\sqrt{2 \pi i \gamma_{1}}} \sin \frac{s x}{\gamma_{1}}\right\rangle\right\rangle
\end{aligned}
$$




$$
\begin{aligned}
= & \left\langle e^{\frac{i}{2}\left(\frac{\alpha_{1}}{\gamma_{1}}\right) t^{2}} f(t) \sin \frac{s t}{\gamma_{1}}, \overrightarrow{\mathrm{F}_{c}}\left(e^{\frac{i}{2}\left(\frac{\alpha_{1}}{\gamma_{1}}\right)(2 t x)} g(x)\right)(t)\right\rangle \\
& +\left\langle e^{\frac{i}{2}\left(\frac{\alpha_{1}}{\gamma_{1}}\right) t^{2}} f(t) \cos \frac{s t}{\gamma_{1}}, \overrightarrow{\mathrm{F}_{s}}\left(e^{\frac{i}{2}\left(\frac{\alpha_{1}}{\gamma_{1}}\right)(2 t x)} g(x)\right)(t)\right\rangle .
\end{aligned}
$$

This completes the proof of the theorem.

Theorem 2.5. Let $f \in \mathcal{E}^{\prime}(\mathrm{R})$ then we have

$$
\left(\overrightarrow{\mathrm{F}}_{s} \sin (u t) f(t)\right)(s)=\frac{e^{-\frac{i}{2}\left(\alpha_{2} u^{2} \gamma_{1}\right)}}{2} e^{-i \alpha_{2} s u}\left(\overrightarrow{\mathrm{F}_{c}} f\right)\left(s+\gamma_{1} u\right) .
$$

Proof. Let $f \in \mathcal{E}^{\prime}(\mathrm{R})$ then

$$
\begin{aligned}
\left(\overrightarrow{\mathrm{F}_{s}} \sin (u t) f(t)\right) & (s)=\frac{e^{\frac{i}{2}\left(\frac{\alpha_{2}}{\gamma_{1}}\right) s^{2}}}{\sqrt{2 \pi i \gamma_{1}}}\left\langle\sin (u t) f(t), e^{\frac{i}{2}\left(\frac{\alpha_{1}}{\gamma_{1}}\right) t^{2}} \sin \left(\frac{s}{\gamma_{1}} t\right)\right\rangle \\
& =\frac{e^{\frac{i}{2}\left(\frac{\alpha_{2}}{\gamma_{1}}\right) s^{2}}}{\sqrt{2 \pi i \gamma_{1}}}\left\langle f(t), e^{\frac{i}{2}\left(\frac{\alpha_{1}}{\gamma_{1}}\right) t^{2}} \sin (u t) f(t) \sin \left(\frac{s}{\gamma_{1}} t\right)\right\rangle .
\end{aligned}
$$

Using the identity

$$
2 \sin x \sin y=\cos (x-y)-\cos (x+y),
$$

(13) becomes

$$
\begin{aligned}
\left(\overrightarrow{\mathrm{F}_{s}} \sin (u t) f(t)\right) & (s) \\
& =\frac{e^{\frac{i}{2}\left(\frac{\alpha_{2}}{\gamma_{1}}\right) s^{2}}}{\sqrt{2 \pi i \gamma_{1}}}\left\langle f(t), e^{\frac{i}{2}\left(\frac{\alpha_{1}}{\gamma_{1}}\right) t^{2}} \frac{\left(\cos \left(\frac{s}{\gamma_{1}} t-u t\right)-\cos \left(\frac{s}{\gamma_{1}} t+u t\right)\right)}{2}\right\rangle .
\end{aligned}
$$

Calculations on (14) together with the equation

$$
e^{\frac{i}{2}\left(\frac{\alpha_{2}}{\gamma_{1}}\right) s^{2}}=e^{-\frac{i}{2}\left(\alpha_{2} u^{2} \gamma_{1}\right)} \cdot e^{\frac{i}{2}\left(\frac{\alpha_{2}}{\gamma_{1}}\right)\left(s-u \gamma_{1}^{2}\right)} \cdot e^{i \alpha_{2} u s}
$$

and that

$$
e^{\frac{i}{2}\left(\frac{\alpha_{2}}{\gamma_{1}}\right) s^{2}}=e^{-\frac{i}{2}\left(\alpha_{2} u^{2} \gamma_{1}\right)} \cdot e^{\frac{i}{2}\left(\frac{\alpha_{2}}{\gamma_{1}}\right)\left(s+u \gamma_{1}^{2}\right)} \cdot e^{-i \alpha_{2} u s},
$$

when employed to (14), give

$$
\left(\overrightarrow{\mathrm{F}}_{s} \sin (u t) f(t)\right)(s)=\left(\frac{1}{2} e^{-\frac{i}{2}\left(\alpha_{2} u^{2} \gamma_{1}\right)} \cdot e^{i \alpha_{2} s u}\right) \frac{e^{\frac{i}{2}\left(\frac{\alpha_{2}}{\gamma_{1}}\right)\left(s-u \gamma_{1}\right)^{2}}}{\sqrt{2 \pi i \gamma_{1}}}
$$




$$
\begin{aligned}
& \times\left\langle f(t), e^{\frac{i}{2}\left(\frac{\alpha_{1}}{\gamma_{1}}\right) t^{2}} \cos \left(\frac{s-u \gamma_{1}}{\gamma_{1}}\right) t\right\rangle \\
& -\left(\frac{1}{2} e^{-\frac{i}{2}\left(\alpha_{2} u^{2} \gamma_{1}\right)} e^{-i \alpha_{2} s u}\right) \\
& \times \frac{e^{\frac{i}{2}\left(\frac{\alpha_{2}}{\gamma_{1}}\right)\left(s-u \gamma_{1}\right)^{2}}}{\sqrt{2 \pi i \gamma_{1}}}\left\langle f(t), e^{\frac{i}{2}\left(\frac{\alpha_{1}}{\gamma_{1}}\right) t^{2}} \cos \left(\frac{s+u \gamma_{1}}{\gamma_{1}}\right) t\right\rangle .
\end{aligned}
$$

which produces our desired result.

Theorem 2.6. Let $f \in \mathcal{E}^{\prime}(\mathrm{R})$ then

$$
\left(\overrightarrow{\mathrm{F}_{c}} \cos (u t) f(t)\right)(s)=\frac{e^{-\frac{i}{2}\left(\alpha_{2} u^{2} \gamma_{1}\right)}}{2}\left(\begin{array}{c}
e^{-i \alpha_{2} s u}\left(\overrightarrow{\mathrm{F}_{s}} f\right)\left(s+\gamma_{1} u\right)+ \\
e^{i \alpha_{2} s u}\left(\overrightarrow{\mathrm{F}_{s}} f\right)\left(s-\gamma_{1} u\right)
\end{array}\right)
$$

Proof of this theorem is quite analogous to that of Theorem 2.5, thus avoided.

\section{References}

[1] S.K.Q. Al-Omari, D. Loonker, P.K. Banerji, S.L. Kalla, Fourier sine(cosine) transform for ultradistributions and their extensions to tempered and ultraboehmian spaces, Integ. Trans. Spl. Funct., 19, No. 6 (2008), 453-462.

[2] S.K.Q. Al-Omari, The generalized Stieltjes and Fourier transforms of certain spaces of generalized functions, Jord. J. Math. Stat., 2, No. 2 (2009), $55-66$.

[3] S.K.Q. Al-Omari, On the distributional Mellin transformation and its extension to Boehmian spaces, Int. J. Contemp. Math. Sciences, 6, no. 17 (2011), 801-810.

[4] R.S. Pathak, Integral Transforms of Generalized Functions and their Applications, Gordon and Breach Science Publishers, Australia-Canada-IndiaJapan (1997).

[5] S.K.Q. Al-Omari, A. Kilicman, On diffraction Fresnel transforms for Boehmians, Abstract and Applied Analysis, 2011, Article ID 712746.

[6] P.K. Banerji, S.K. Alomari, L. Debnath, Tempered distributional Fourier sine(cosine) transform, Integ. Trans. Spl. Funct., 17, No. 11 (2006), 759768. 
[7] A.H. Zemanian, Generalized Integral Transformation, Dover Publications Inc., New York; First Published by Interscience Publishers, New York (1968). 
